\title{
PETECHIAE WITH CYANOSIS IN THE NEWBORN
}

\author{
BY
}

\author{
N. C. ELPHINSTONE, M. BRENDA MORRIS and SIMON YUDKIN \\ From Whittington Hospital, London
}

(RECEIVED FOR PUBLICATION OCTOBER 10, 1953)

In the past two or three years our attention has been drawn to a condition in newborn babies which, although not particularly uncommon, does not seem to be widely known and which may give rise to needless anxiety.

The doctor is usually called to see a baby which is born spontaneously but after birth appears to be grossly cyanosed. A nurse has usually put the baby into an oxygen tent. On two or three occasions a general practitioner who has delivered the baby or has been called in by the midwife has brought the baby to the hospital as an emergency.

When examined the baby is well and lusty. Most of the body is pink but the face and the head and part of the neck are dark purple. This colour sometimes also involves the lips and tongue. On looking more closely at the skin of the face there is seen to be a multitude of almost confluent petechiae but the cyanosis extends to the lips and tongue where there are no petechiae and sometimes a little beyond the edge of the petechial area. Occasionally there is also a sub-conjunctival haemorrhage.

The petechiae change colour during the next few days and have usually gone by the tenth day. The accompanying cyanosis lasts only for a day or two. The rest of the clinical examination is usually completely normal.

Sometimes, but not more frequently than in other babies, the hands and feet of these babies show 'peripheral' i.e., local, cyanosis with poor circulation.

No treatment is necessary.

These babies prompted us to look more carefully at the incidence of petechiae on the skin of the newborn and a series of babies was examined. It soon became obvious that many petechiae were missed unless very carefully looked for. A series of 98 babies was therefore examined with great care within a few hours of birth in bright daylight; a glass slide was used to differentiate between minute blood vessels and petechial haemorrhages.

Petechiae were searched for on the scalp and face and on the rest of the body.
In about a quarter of the babies presenting by the vertex there were petechiae limited to a small area of the scalp only, but in about a further half of the babies there were petechiae elsewhere in addition, usually on the forehead, cheeks, chin or neck. Only very occasionally were they found in other parts of the body and then they were much less numerous than on the face and neck.

In babies born by breech delivery the petechiae if present were on the presenting buttock.

In this series there were five babies born by Caesarean section. In none of these had the head engaged in the pelvis. Four had no petechiae and one had a single petechial haemorrhage on the scalp.

Since this series of babies was examined a baby, who was born by Caesarean section, has been seen with gross local cyanosis and petechiae involving the face and to a lesser degree the scalp and neck. This baby's head was delivered with difficulty with the aid of forceps and was held in the uterine incision, with the face presenting for some 30 to 60 seconds.

In only 34 of the babies were no petechiae found.

An attempt was made to see whether subconjunctival haemorrhages were correlated with the degree of petechiae. Of 74 babies whose eyes were examined, 10 had subconjunctival haemorrhages and in eight of these there were petechiae elsewhere than on the scalp.

Analysis of various factors showed no correlation between the incidence of petechiae and maternal history, the type of delivery, length of labour, anaesthetic and the condition of the baby at birth. However, they were more common in male than in female babies and tended to be more extensive in the heavier babies.

Prothrombin time and platelet counts were normal. One baby had petechiae sparsely distributed over the whole of the body, accompanied by a platelet count of $15,000 / \mathrm{c} . \mathrm{mm}$. The mother also had thrombocytopenia and this was considered to be a case of thrombocytopenic purpura of the newborn.

This condition of petechiae with accompanying 
cyanosis seems to us to resemble traumatic cyanosis in older children and adults and it may be produced by an analogous mechanism.

\section{Conclusions and Summary}

Petechiae on the neck and head of the newborn baby (born by the vertex) are not uncommon, especially in large babies, and some petechiae were found in almost two-thirds of the babies examined.

When profuse they are usually accompanied by local cyanosis, an appearance which may cause needless anxiety. However, the baby is otherwise well and there is no central cyanosis.
Petechiae elsewhere on the body are rare in this condition and usually only sparse.

In babies born by the breech a similar condition occurs on the buttocks.

The condition seems to resemble traumatic cyanosis and may be produced by a similar mechanism, occurring during delivery of the presenting part.

We would like to thank Dr. Simpson, of the Pathology Department, for carrying out the haematological tests and the nursing staff of the Maternity Department who were so prompt in drawing our attention to the condition once it had been recognized. 